\title{
Scaling Laws for Modeling Large Superconducting Solenoids*
}

\author{
M. A. Green and A. D. McInturff
}

\begin{abstract}
The neutrino factory cooling system will consist of a long series of superconducting solenoids with a warm bore of 1.2 meters. In order to minimize the cost of the 200 to 300-meter long solenoid muon-cooling channel, the solenoids must be fabricated so that their mass is minimized. This report discusses how one can model the stress, strain and quench behavior of these large solenoid sections by building one-third to one-half scale models of the magnets. The cost of building and engineering the scale model magnets is a small fraction of the cost of fabricating a full-scale magnet section. This report discuses the limitations of the scaling approach as well as the types of superconducting solenoids for which the modeling technique is suitable.
\end{abstract}

\section{INTRODUCTION}

The muon collider and neutrino factory will have hundreds of meters of large superconducting solenoids to carry pion and muon beams. These solenoids will be located in the phase rotation section downstream from the target, the muon cooling sections and some of the pre-acceleration sections of the machine[1,2]. Figure 1 shows a typical Super FOFO cell, with a flux reversal in the liquid hydrogen absorber. The large superconducting coils must fit around the $200 \mathrm{MHz}$ RF cavity. This means the warm bore diameter of the large solenoid is about 1.2 meters. The role of the liquid hydrogen absorbers is to reduce the longitudinal and transverse momentum of the muons. The RF cavity restores momentum in the longitudinal direction without introducing much transverse momentum.

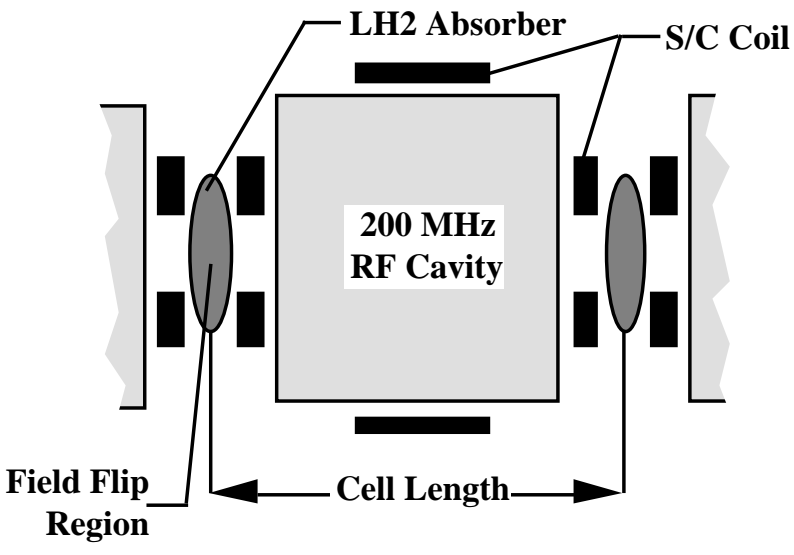

Figure 1. Typical Super FOFO Muon Cooling Section

Manuscript received 18 September 2000. The authors are from Lawrence Berkeley National Laboratory, Berkeley CA 94720 , USA

* This Research is supported by the Office of Science US Department of Energy under contract number DE-AC0376SF00098
Since there are many of these solenoid sections, it is desirable to minimize their cost by minimizing the material in the solenoid magnet sections. This means that the superconducting coil will operate above the cryogenically stability limit. The solenoidal coil will carry the magnetic stress and it must quench safely

This report describes the rules for building working scale models of superconducting solenoids that can be used for a variety of applications from high-energy physics to medical imaging. This report describes the types of solenoids that can be modeled and those that can not be modeled. Scaling rules for stress and quench protection are presented.

\section{WHY MODEL S UPERCONDUCTING S OLENOIDS?}

The short answer as to why one would build working models of superconducting solenoids is to save money during the magnet development process. The following cost equation explains why modeling can be attractive[3]:

$$
C(M \$)=0.53 M(\text { tons })^{0.75}
$$

where $\mathrm{C}$ is the cost of the magnet and its cryostat in millions of US dollars, and $\mathrm{M}$ is the magnet coil and cryostat mass in metric tons. From equation 1 it is clear that a half-scale model that has about one-seventh of the mass of a full-scale model will cost about 23 percent of the cost of the full-scale magnet. A one-third scale model will have about onetwentieth of the mass of the full scale model and will cost about 11 percent of the cost of the full-scale magnet. It should be noted that the cost equation given above includes engineering as well as fabrication.

Before a magnet development program uses scale models, it should be pointed out that there are certain limitations to scaling solenoid magnets. Scaling appears to be a useful under the following circumstances[4]: 1) The size of the largest coils in the full-scale solenoid system should be over 1-meter in diameter. The thickness of the large diameter coils should be determined by stress in the coils and the coil support structure. 2) The cross-section area of the stress carrying parts of the coil (the conductor matrix material and any support structure for hoop forces) should be at least five times the area of the superconductor. This means that the stresses in the model conductor will be similar to the stress in the full-scale coil and its support structure. 3) The crosssection area of the coil insulation should be less than 15 percent of the total cross-section area of the coil and support structure. Magnet coil insulation has a much lower elastic modulus and a lower yield stress. One wants the crosssectional area of both the full scale magnet and the model 
magnet to be dominated by conductor matrix material and support structure. 4) The void fraction of the coil and its hoop force support structure should be small. Voids in the coil and support structure contribute to a redistribution of the coil stress. One wants the stress in the model magnet to be distributed in the same way that it is in the full-scale magnet. 5) Scale model magnets may also be useful for large MHD type dipoles where the full-scale model superconductor has a copper to $\mathrm{S} / \mathrm{C}$ ratio of at least six. The construction of model magnets appears to be a useful technique for large potted solenoids, such as detector magnets and the solenoids used for muon cooling. Other applications might include solenoids used for full body MRI and magnetic separation.

\section{MODELING S TRESS IN S IMPLE SOLENOIDS}

The simplest type of solenoid is an infinite solenoid or a section of an infinite solenoid. In this type of solenoid, the peak induction in the coil and the central induction are the same. The induction outside the coil is zero. The induction $\mathrm{B}_{\mathrm{z}}$ in an infinite solenoid is given as follows:

$$
B_{z}=\mu_{0} n I
$$

where $\mathrm{nI}$ is the number of ampere turns per meter and $\mu_{0}$ is the permeability of air. The current density in the winding $\mathbf{J}$ is uniform along the length of the solenoid. For a given coil thickness $t$, the value of $\mathrm{J}$ is as follows:

$$
J=\frac{n I}{t}=\frac{B_{z}}{\mu_{0} t} .
$$

Since the magnet behaves as a magnetic pressure vessel, the average stress in the coil $\sigma$ is as follows:

$$
\sigma=\frac{P_{m} R}{t}=\frac{B_{z}^{2}}{2 \mu_{0}} \frac{R}{t},
$$

where $\mathrm{Pm}$ is the magnetic pressure and $\mathrm{R}$ is the average coil radius.

For a thin infinite solenoid where $t<<R$, the peak potential stress or BRJ stress is two times the average stress. The BJR stress would be the peak stress in the coil, if the region of the coil at the peak field point were mechanically de-coupled from the rest of the coil. The BJR stress is not real for solenoids, with conductors that are mechanically coupled. Thick coils that are not well coupled mechanically (cryogenically stable coils with flexible spacers) will have a peak stress approaching the BJR stress. $R$ in this case is the radius of the point where the peak induction $B$ (parallel to the solenoid axis) occurs. $\mathrm{J}$ is the coil conductor current density.

The stored energy per unit length E can be calculated with the following expression:

$$
E=\frac{B_{z}^{2}}{2 \mu_{0}} \pi R^{2}
$$

By comparing equations 4 and 5 one can see that there is a direct correlation between the stress in the winding and the stored energy per unit length. As a result, the following scaling expressions are true:

$$
\begin{aligned}
& \sigma=\frac{E}{\pi R t}, \\
& t=\frac{B_{z}^{2}}{2 \mu_{0}} \frac{R}{\sigma}, \\
& \text { and } J=\frac{\sigma}{B_{z} R} .
\end{aligned}
$$

Equations 6, 7, and 8 suggested that for a given design stress in the winding, the thickness of the coil t must go up as the induction $\mathrm{B}$ squared and the coil radius $\mathrm{R}$.

From the equations above, the scaling laws for large solenoid magnets based on stress can be established. When one scales the magnet, one wants to operate the scale model at the same level of stress as the full-scale magnet. This means that the physical thickness of the conductor in the coil $\mathrm{t}$ is proportional to the average coil radius $\mathrm{R}$. For a given level of stress in the coil conductor $\sigma$, the current density in the coil conductor $\mathrm{J}$ must be inversely proportional to the coil average radius $\mathrm{R}$. (Note: the support structure stress is correct if its thickness also scales with the conductor thickness.) The bottom line is that the conductor and support structure dimensions must scale with average coil radius.

Two factors affect coil scaling at constant stress. The first is the normal metal to superconductor ratio in the conductor. The area of the superconductor does not scale. If the full-scale coil has 10 percent of its cross-section superconductor, a halfscale model must have 20 percent of its cross-sectional area superconductor in order for the coil to reach its design induction with the same margin along the load line as the full-scale coil. For coils made with $\mathrm{Nb}$ - $\mathrm{Ti}$ conductor, the modulus of the coil is not greatly affected by the normal metal to superconductor ratio. $\mathrm{Nb}_{3} \mathrm{Sn}$ coils are different.

The second factor in coil scaling is the coil insulation. In general, one can not reduce the thickness of the insulation as one scales the coil and conductor dimensions. The coil insulation may have a greater effect on scaling than does the superconductor. The effect is small provided the conductor thickness is scaled with $\mathrm{R}$ and the insulation is less than 20 percent of the total coil area in the full-scale magnet coil.

\section{SCALING OF THE CRYOSTAT}

To first order, the metal portions of the cryostat vacuum vessel scale as the metal portions of the superconducting coils. One can prove this by looking at the equations for calculating the thickness for a solenoid warm bore tube $t_{1}$ and the thickness of the outer vacuum vessel $t_{2}$. The following expressions can be used to calculate $\mathrm{t}_{1}$ and $\mathrm{t}_{2}[5]$ : 


$$
\begin{aligned}
& t_{1}=2 \frac{P_{o} R}{\sigma_{u}} \\
& \text { and } t_{2}=1.43\left[\frac{P_{o} L R^{1.5}}{E}\right]^{0.4}
\end{aligned}
$$

where $P_{o}$ is the pressure outside of the vacuum vessel; $\sigma_{u}$ is the ultimate stress for the cryostat vacuum vessel material, $\mathrm{L}$ is the length of the vacuum vessel, and $\mathrm{E}$ is the modulus of elasticity of the cryostat vacuum can material.

From equation 9, one can see that $t_{1}$ is proportional to $\mathrm{R}$. From equation 10 , one can see the $t_{2}$ is proportional to $R$ provided $\mathrm{L}$ scales along with $\mathrm{R}$. The only thing that does not scale with $\mathrm{R}$ is the space needed for the cryostat insulation.

\section{THE EFFECT OF QUENCH PROTECTION ON SCALING}

The second design condition one must consider when building a scale model of a superconducting solenoid is the quench protection of the magnet. The quench protection criterion is the hot spot temperature $\mathrm{T}_{\mathrm{M}}$ for the coil. This temperature in generally limited by the insulation on the wire, thus the maximum design value for $\mathrm{T}_{\mathrm{M}}=400 \mathrm{~K}$. The hot spot temperature criteria is governed by integrated current density function which in turn is governed by the specific heat over resistivity integral from $4 \mathrm{~K}$ to $\mathrm{T}_{\mathrm{M}}$. The integrated current density function $\mathrm{F}^{*}$ takes the following form[6,7]:

$$
F^{*}=\int_{4}^{T_{M}} \frac{C(T)}{\rho(T)} d T=\frac{r+1}{r} \int_{t=0}^{\infty} J^{2}(t) d t,
$$

where $\mathrm{C}$ is the volume specific heat; $\rho$ is the resistivity of the matrix material; $r$ is the normal metal to superconductor ratio for the conductor; $\mathrm{J}$ is superconductor plus matrix current density; $\mathrm{T}$ is temperature; and $\mathrm{t}$ is time. $\mathrm{F}^{*}$ relates the physical properties of the conductor to the current density integral with time. $\mathrm{F}^{*}$ is a function of the conductor matrix material and $\mathrm{T}_{\mathrm{M}}$. For example $\mathrm{F}^{*}$ for $\mathrm{T}_{\mathrm{M}}=400 \mathrm{~K}$ for copper $R R R=100$ is $17 \times 10^{16} \mathrm{~A}^{2} \mathrm{~m}^{-4} \mathrm{~s}$, and $\mathrm{F}^{*}$ for $\mathrm{T}_{\mathrm{M}}=400 \mathrm{~K}$ for aluminum $R R R=1000$ is $7.6 \times 10^{16} \mathrm{~A}^{2} \mathrm{~m}^{-4} \mathrm{~s}$.

The value of $\mathrm{F}^{*}$ determines the exponential $\mathrm{L} / \mathrm{R}$ time constant $\tau$ for a safe quench and it determines the $\mathrm{EJ}^{2}$ limit for the magnet section, once one knows the coil design current I and the discharge voltage during a quench $\mathrm{V}$. The values of the safe quench L/R time constant $\tau$, and the magnet $\mathrm{EJ}^{2}$ limit can be calculated using the following expressions:

$$
\begin{aligned}
& \tau=\frac{2 F^{*}\left(T_{M}\right)}{J_{o}{ }^{2}} \frac{r}{r+1} \\
& \text { and } E_{o} J_{o}{ }^{2}=V I F *\left(T_{M}\right) \frac{r+1}{r}
\end{aligned}
$$

where $E_{o}$ is the stored energy of the magnet section at its design current $\mathrm{I}$ and $\mathrm{J}_{\mathrm{o}}$ is the matrix plus superconductor current density at a magnet design current I.
The $\mathrm{EJ}^{2}$ limit shown in equation 13 can be related directly to the average stress $\sigma$ in the coil conductor. This relationship is as follows:

$$
E J^{2}=\frac{2 \pi}{\mu_{0}} \sigma^{2}
$$

Thus as in equation 8 the conductor average stress is related directly to the current density in the conductor. From Equation 14, one can see that quench protection scales as stress scales. The only requirement is that the conductor matrix dimensions must scale as R. To first order, the design current in the conductor I must also scale as R.

Quench-back has been looked at in the context of magnet scaling[8]. Studies in reference 4 indicate that the voltage needed to induce quench-back in a half-scale model coil is almost double that of a full-scale magnet. Quench-back does not appear to scale very well. Large diameter solenoids appear to quench-back easier than small diameter solenoids.

\section{SCALING A 1 METER DIAMETER SOLENOID S YSTEM}

A demonstration of how scaling can be used to model a full sized 1-meter diameter $5 \mathrm{~T}$ solenoid that is part of a string of solenoids is illustrated in Figure 2 and in Table 1.
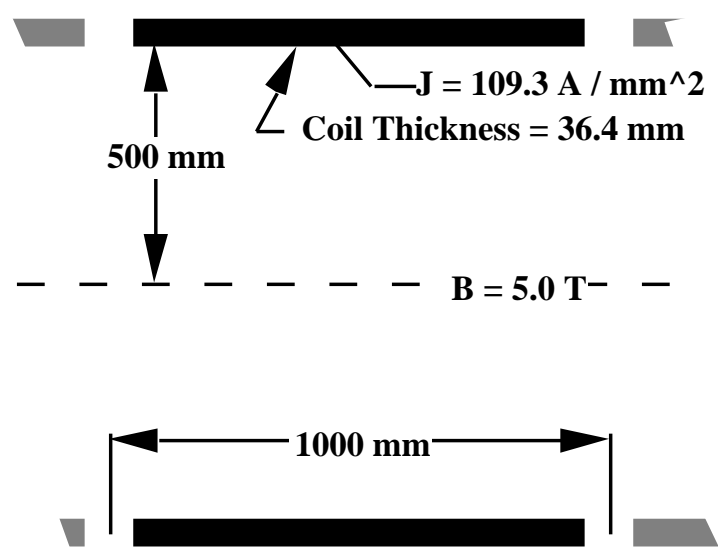

FULL-SCALE MAGNET SECTION

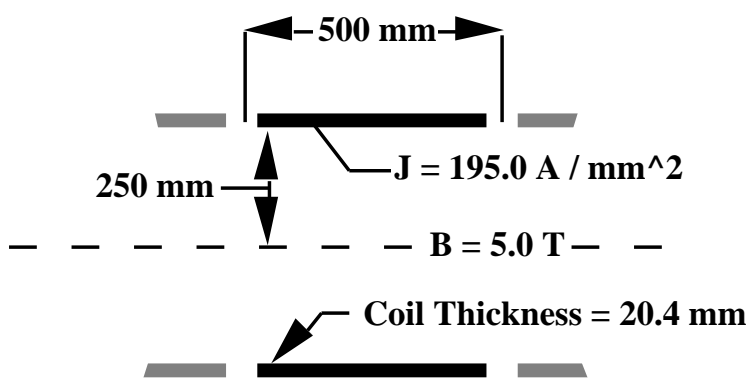

HALF- SCALE MODEL MAGNET

Figure 2 A Schematic Representation of a Full Scale Solenoid Magnet Section and a Half Scale Model of the Section 
TABLE I. A COMPARISON OF S TRESS AND QUENCH BEHAVIOR FOR FULL AND HALF SCALE SOLENOIDS

\section{Parameter}

Full Size Half Size

Magnet Section Physical Parameters

Induction on Axis (T)

Average Radius of Coil (mm)

Coil Section Length $(\mathrm{mm})$

Coil Physical Thickness (mm)

Number of Layers

Number of Turns per Layer

Number of Turns per Coil

Coil Packing Fraction

Superconductor and Coil Parameters

Bare Conductor Size (mm)

Copper to S/C Ratio

Coil Current (A)

Coil Current Density $\left(\mathrm{A} \mathrm{mm} \mathrm{mm}^{-2}\right)$

Matrix Current Density $\left(\mathrm{A} \mathrm{mm}^{-2}\right)$

S/C Current Density $\left(\mathrm{A} \mathrm{mm}^{-2}\right)$

Section Stored Energy (MJ)

Section Self Inductance $(\mathrm{H})$

Magnet Stress and Strain Parameters

Ave. Coil Stress w/o Shell (MPa)

Ave. Coil Strain w/o Shell (\%)

Ave. Coil Stress with Shell (MPa)

Ave. Coil Strain with Shell (\%)

Peak Field BJR Stress (MPa)

Quench Protection Parameters

Safe Quench Time Constant (sec)

Dump Resistance No QB (ohm)

Dump Voltage No QB (V)

$\mathrm{EJ}^{2}$ Limit $\left(\mathrm{A}^{2} \mathrm{~m}^{-4} \mathrm{~J}\right)$

Quench Velocity $\left(\mathrm{m} \mathrm{s}^{-1}\right)$

Time for Coil to Quench (sec)

Self-Protecting w/o QB

$\mathrm{R}$ for $\mathrm{QB}$ from $\mathrm{RRR}=25 \mathrm{Al}$ (ohm)

Quench-Back Voltage (V)

Self-Protecting with QB

Coil Cost Factor (Full Size $=1$ )

The scaling laws say one should scale the superconductor matrix dimensions with $\mathrm{R}$. As a result, the half scale magnet coil is $20.4 \mathrm{~mm}$ compared to the $36.4-\mathrm{mm}$ thick full-scale coils. The $2.2-\mathrm{mm}$ of extra thickness is the layer to layer electrical insulation, which does not scale. There are fewer turns per layer in the half scale coil than there are in the fullscale coil, even though the bare matrix dimensions for the superconductor in the half-scale model conductor are half that of the full-scale model conductor. The reason is that the turn to turn insulation does not scale. As a result, the current in the half-scale model conductor is a little over half of the current in the full-scale conductor. The average stress and BJR peak stresses are about 2 percent higher for the half-scale magnet than for the full-scale magnet. The voltage needed for a safe discharge of the half-scale magnet is about 15 percent larger than is needed for the full-scale magnet. The primary reason for this is the lower copper to superconductor ratio for the half-scale model magnet conductor.
An aluminum shell on the outside of the coil does reduce the stress in the coil. This is true in both the full-scale and half-scale magnets. If the aluminum shell is made from $R R R=25$ aluminum, quench-back can be induced at much lower voltages than are needed to protect the coil in a quench without the aluminum shell. Because both the full scale and the half scale coils go fully normal in a time that is less than the safe quench time constant, they may be self protected without a $R R R=25$ aluminum shell. With the aluminum shell present, quench-back will protect both coils without an external quench protection system[5].

\section{CONCLUDING COMMENTS}

The construction of scale model magnets appears to be a viable way of developing large superconducting solenoid systems. This report demonstrates scaling using a simple solenoid. If one follows the scaling laws correctly, complex solenoid systems can also be modeled, provided all of the solenoids in the system follow the scaling laws. The scalemodel magnet exaggerates both the stress and the quench protection problems compared to the full-scale magnet. The primary reasons for this are the increased percentages of superconductor and insulation in the coils. As a result, if the scale-model magnet operates without training, the full-scale magnet should also perform well. Since magnet training and related phenomena are strain related, modeling the full-scale magnet system with a smaller scale model should permit one to develop coil designs that do not train. The cost of the scale-model solenoid system is a fraction of the cost of a fullscale solenoid magnet system.

\section{REFERENCES}

[1] M. A. Green, Y. Eyssa, S. Kenny, et al, "Superconducting Solenoids for the Muon Collider," IEEE Transactions on Applied Superconductivity 10, No. 1, p 196, (2000)

[2] N. Holltkamp, et al, "A Feasibility Study of a Neutrino Source Based on a Muon Storage Ring" FERMILAB-PUB-00108-E; (2000), submitted to Phys. Rev. ST Accel. Beams

[3] M. A. Green and S. J. St. Lorant, "Estimating the Cost of Large Superconducting Thin Solenoid Magnets," Advances in Cryogenic Engineering 39, p 271, Plenum Press (1993)

[4] M. A. Green, "Design Issues for the Solenoid Magnets for the Neutrino Factory Cooling System," Lawrence Berkeley National Laboratory report LBNL-46357, (2000)

[5] M. A. Green, "High Energy Physics Magnets," Wiley Encyclopedia of Electrical and Electronics Engineering, Vol. 8, Wiley-Interscience Publication, New York (1999)

[6] B. J. Maddock and G. B. James, "Protection and Stabilization of Large Superconducting Coils," Proceedings of the IEE 115, No. 4, p 543 (1968)

[7] P. H. Eberhard et al, "Quenches in Large Superconducting Magnets," MT-6, Proceedings, Bratislava Czechoslovakia, p 654 (1977)

[8] M. A. Green, "Quench Back in Thin Superconducting Solenoid Magnets," Cryogenics 24, p 659 (1984) 